\title{
Studying the complexity of identification of Volterra kernels for the case of a vector input signal of arbitrary dimension
}

\author{
Ekaterina Antipina ${ }^{1, *}$, Emir Tairov ${ }^{1}$, and Evgeniia Markova ${ }^{1}$ \\ ${ }^{1}$ Melentiev Energy Systems Institute SB RAS, 664033 Lermontov St., 130, Irkutsk, Russia
}

\begin{abstract}
The work discusses the technique for constructing an integral model of a nonlinear dynamic system with a vector input based on Volterra polynomials as applied to a section of the steam-water path of the power unit of the Nazarovo power station. The complexity of the applying the technique presented in the work is analyzed, and the number of initial data required to build a mathematical model in the case of a vector input disturbance with an arbitrary dimension is calculated.
\end{abstract}

Keywords. Volterra polynomials, identification, dynamic system, initial data.

\section{Introduction}

Various methods and algorithms are used to construct mathematical models of energy objects. For example, in [1] the authors use associative search algorithms and imprecise algorithms to identify the state of energy objects. The method of control equations was used to estimate the state of electric power systems [2]. Artificial neural networks are used to solve electric power problems [3]. The authors of [4] build a mathematical model using the methods of J. Box and G. Jenkins to study the operation of the combustion unit.

In this work, the technique of constructing mathematical models using the apparatus of Volterra integro-power polynomials is considered. This apparatus is universal and also convenient for describing such dynamic processes, the application to which of other methods is extremely difficult or practically impossible, for example, during the operation of energy objects. Methods for constructing a model of a segment of a steam-water path of a power unit of Nazarovo power station based on this apparatus were considered in $[5,6]$. In these papers, scalar models (with one input and one output) were considered.

This paper presents the development of an integral model for the case of a two-dimensional input: disturbances in the flow rates of steam and cooling water to a condensing unit. The analysis of the required volume of data, got from test experiments, to construct a model of a dynamic system with a vector input of arbitrary dimension is carried out.

\section{Description of the technique}

The considered mathematical apparatus is used to describe objects of the "input-output" type, they are also called objects of the "black box" type (Fig. 1). In this case, a dynamic object can be represented as a finite segment of the Volterra integro-power series [7]

$$
\begin{gathered}
y(t)=\sum_{m-1}^{N} \sum_{1 \leq i_{1} \leq \ldots \leq i_{m} \leq p} f_{i_{1} \ldots i_{m}}(t), t \in[0, T], \\
f_{i_{1} \ldots i_{m}}(t)=\int_{0}^{t} \ldots \int_{0}^{t} K_{i_{1} \ldots i_{m}}\left(s_{1}, \ldots, s_{m}\right) \prod_{j=1}^{m} x_{j}\left(t-s_{j}\right) d s_{j} . \\
\stackrel{x_{p}(t)}{\stackrel{x_{1}(t)}{\longrightarrow}} \stackrel{y(t)}{\longrightarrow}
\end{gathered}
$$

Fig. 1. Block diagram of a dynamic system of the "black box" type.

Here the input signal $x(t)=\left(x_{1}(t), \ldots, x_{p}(t)\right)^{T}$ is a vector function of time; the response $y(t)$ is a scalar function of time; Volterra kernels $K_{i_{1} \ldots i_{m}}$ are symmetric in the variables $s_{1}, \ldots, s_{m}$, that correspond to the coinciding indices $i_{1}, \ldots, i_{m}$. In (1), (2) the system is stationary, i.e. its dynamic characteristics do not change over the entire time of the transient process. In other words, the kernels $K_{i_{1} \ldots i_{m}}$ are time-independent.

In contrast to the scalar case, in the case of a vector input signal, terms appear in which the Volterra kernels are non-symmetric functions concerning the variables $s_{1}, \ldots, s_{m}$.

Construct a model of the form (1), (2) means to solve the identification problem. The technique of restoring symmetric kernels, based on the supply of test signals in the form of combinations of Heaviside functions, is described in detail in the monograph [8]. The identification of non-symmetric kernels using this technique is described in [9]. 
Consider the developed technique in relation to modeling a specific heat and power object to get recommendations for building a vector model.

\section{Description and analysis of the results of using test experiments}

Consider a segment of the steam-water path of the power unit of Nazarovo power station for a vector input signal containing two components (Fig. 2). This segment includes a condenser K type $80-\mathrm{KTC}-1$. Scalar models have already been constructed for it [6]. Therefore, to construct a vector model with two input signals, it is sufficient to find the values of the non-symmetric kernel.

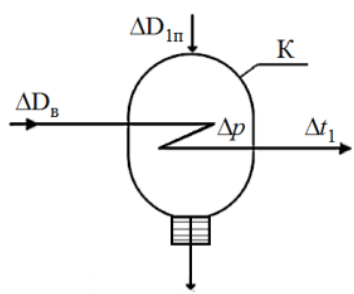

Fig. 2. Schema of segment of the steam-water path of the power unit.

It is assumed that the input is a simultaneous supply of water flow $\Delta D_{w}$ and steam flow $\Delta D_{1 v}$. Their initial values are $D_{0 w}=11562.2 \mathrm{~kg} / \mathrm{s}$ and $D_{0 v}=45.51 \mathrm{~kg} / \mathrm{s}$. Depending on the choice of the output signal, we get two models: the output signal is the pressure deviation $\Delta p$ in the condenser in one model and the deviation of the cooling water temperature $\Delta t_{1}$ at the outlet of the condenser in the other model.

To solve the identification problem, it is necessary to obtain the required number of initial data to determine the values of the non-symmetric kernel. The initial data are sets of input and output signals on a mesh determined by the chosen numerical method. The data collection is carried out by analogy with the work [6], with the help of the "P150" Software Package, which is a development of the model of the power unit of the Irkutsk Central Heating and Power Plant (CHPP-10) [10]. The mathematical model of the power unit of the Irkutsk CHPP-10 includes about one hundred algebraicdifferential and five hundred algebraic equations and closing relations.

According to the technique [8], we input signals of the form:

$$
\begin{aligned}
& \left\{\begin{array}{l}
\Delta D_{w}(t)=\alpha \cdot \mathrm{e}(t), \\
\Delta D_{1 v_{\omega}}(t)=\beta \cdot(\mathrm{e}(t)-\mathrm{e}(t-\omega)), t \in[0, T], 0 \leq \omega \leq t,
\end{array}\right. \\
& \left\{\begin{array}{l}
\Delta D_{w_{\omega}}(t)=\alpha \cdot(\mathrm{e}(t)-\mathrm{e}(t-\omega)), \\
\Delta D_{1 v}(t)=\beta \cdot \mathrm{e}(t), t \in[0, T], 0 \leq \omega \leq t .
\end{array}\right.
\end{aligned}
$$

Here $\mathrm{e}(t)$ is the Heaviside function, $\alpha, \beta$ are the amplitudes of the disturbing influences.

The graphs shown in Fig. 3 correspond to (3), and the graphs shown in Fig. 4 correspond to (4) at $\alpha=0.3 D_{0 w}, \beta=0.3 D_{0 v}$. In the figures, the number 1 indicates the graphs for $t=88, \omega=8$, number $2-$ for $t=88, \omega=48$, number $3-$ for $t=120, \omega=80$, number $4-$ for $t=120, \omega=120$.
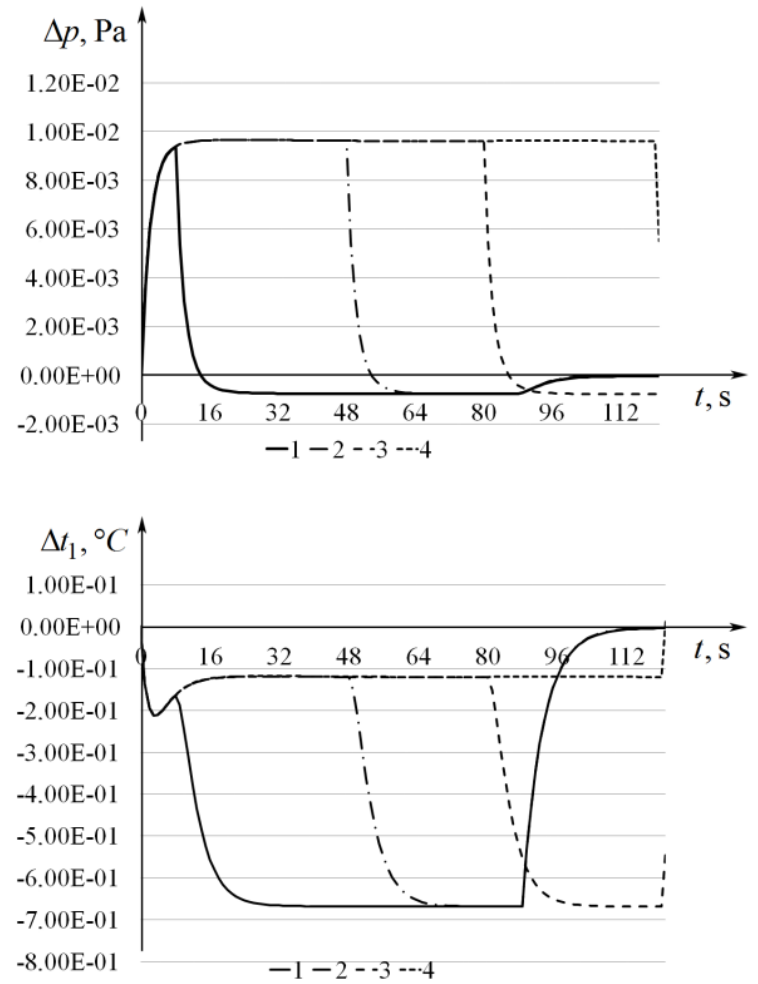

Fig. 3. The pressure deviation $\Delta p$ in the condenser and the deviation of the cooling water temperature $\Delta t_{1}$ at the outlet of the condenser for input signal (3).
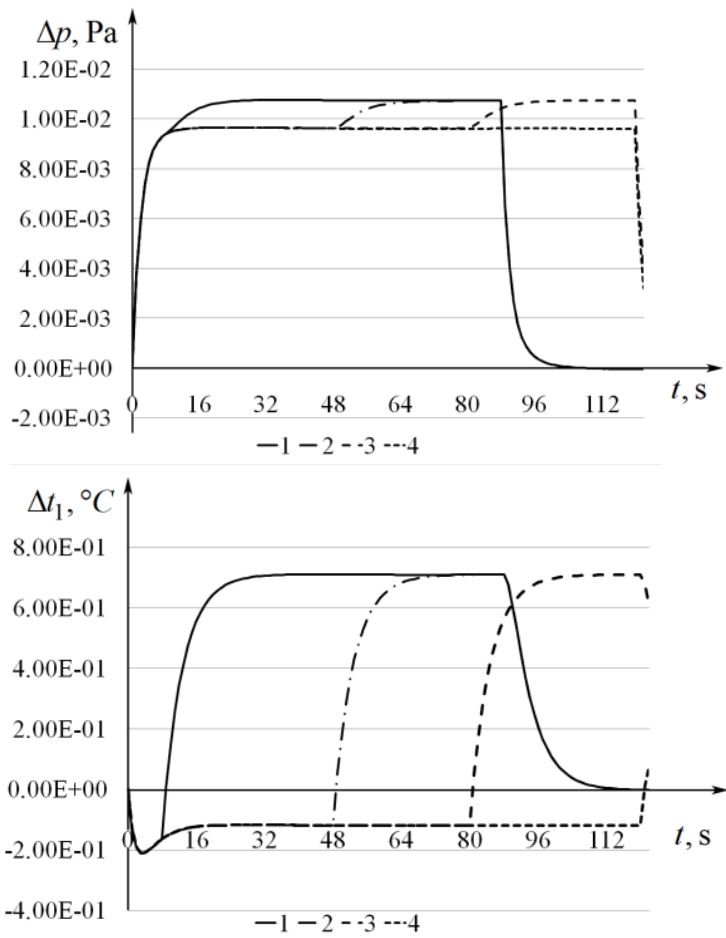

Fig. 4. The pressure deviation $\Delta p$ in the condenser and the deviation of the cooling water temperature $\Delta t_{1}$ at the outlet of the condenser for input signal (4). 
It can be seen from the graphs that the disturbances presented here act in opposition to each other while the change in steam flow has a greater impact on the output than the water flow.

This type of graphs is consistent with the application of our chosen technique. It can be seen that these system responses either reach a new stationary state or return to the previous one. This is important for applying this methodology. We also note that with the given input actions $\Delta D_{w}, \Delta D_{1 v}$, the values of $\Delta p$ and $\Delta t_{1}$ are stabilized with an accuracy of $\delta=10^{-5}$ for $T \geq 23$ (s) and with an accuracy of $\delta=10^{-6}$ for $T \geq 31$ (s). As a result, we find that the duration of transients covers the time range $t \in[0, T]$, where $T \approx 35$ (s).

In this section, we received recommendations for choosing the length of the time interval for given input disturbances. Further, we will consider the complexity of building such a model for an arbitrary number of input disturbances.

\section{Analysis of the complexity of the technique implementation in the case of a vector input disturbance of arbitrary dimension}

Let the vector function of the input signals have an arbitrary dimension equal to $p$, i.e.

$x(t)=\left(x_{1}(t), \ldots, x_{p}(t)\right)^{T}$. Consider a segment of the

Volterra series (1) for $N=2$ and $N=3$.

Let $N=2$, then (1), (2) takes the form

$$
\begin{gathered}
\sum_{i=1}^{p} \int_{0}^{t} K_{i}(s) x_{i}(t-s) d s+ \\
+\sum_{i=1}^{p} \int_{0}^{t} \int_{0}^{t} K_{i i}\left(s_{1}, s_{2}\right) x_{i}\left(t-s_{1}\right) x_{i}\left(t-s_{2}\right) d s_{1} d s_{2}+ \\
+\sum_{i=2}^{p} \sum_{j=1}^{i-1} \int_{0}^{t} \int_{0}^{t} K_{j i}\left(s_{1}, s_{2}\right) x_{j}\left(t-s_{1}\right) x_{i}\left(t-s_{2}\right) d s_{1} d s_{2}=y(t),
\end{gathered}
$$

We denote

$$
\begin{gathered}
\int_{0}^{t} K_{i}(s) x_{i}(t-s) d s=V_{i} K_{i}, \\
\int_{0}^{t} \int_{0}^{t} K_{i i}\left(s_{1}, s_{2}\right) x_{i}\left(t-s_{1}\right) x_{i}\left(t-s_{2}\right) d s_{1} d s_{2}=V_{i i} K_{i i}, \\
\int_{0}^{t} \int_{0}^{t} K_{j i}\left(s_{1}, s_{2}\right) x_{j}\left(t-s_{1}\right) x_{i}\left(t-s_{2}\right) d s_{1} d s_{2}=V_{j i} K_{j i},
\end{gathered}
$$

then (5) takes the form

$$
\sum_{i=1}^{p} V_{i} K_{i}+\sum_{i=1}^{p} V_{i i} K_{i i}+\sum_{i=2}^{p} \sum_{j=1}^{i-1} V_{j i} K_{j i}=y(t) .
$$

The problem of calculating the number of necessary input data for building a model consists of two stages. At the first stage, the number of input signals for solving the decomposition problem is determined. At the second stage, a numerical method is chosen and, depending on the mesh division, we count the number of equations to identify each kernel.
Move on to the first stage. To isolate the terms containing symmetric kernels, we supply a series of signals with amplitudes $\alpha_{1_{(i)}}$ and $\alpha_{2_{(i)}}$ (we assume $\alpha_{(j)}$ equal to zero for $j \neq i, j=\overline{1, p}$ ), where $i=\overline{1, p}$. In this case, the condition $\alpha_{1_{(i)}}+\alpha_{2_{(i)}}=0$ must be satisfied [10]. As a result, we obtain a system of algebraic equations

$$
\begin{aligned}
& \alpha_{1_{(i)}} \cdot V_{i} K_{i}+\alpha_{1_{(i)}}^{2} V_{i i} K_{i i}=y^{0, \ldots 0, \alpha_{1_{(i)}}, 0, \ldots 0}(t), \\
& \alpha_{2_{(i)}} \cdot V_{i} K_{i}+\alpha_{2_{(i)}}^{2} V_{i i} K_{i i}=y^{0, \ldots 0, \alpha_{2_{(i)}}, 0, \ldots 0}(t),
\end{aligned}
$$

Thus, the number of all series of signals for the terms containing symmetric kernels is $2 p$.

Solving (7), we get

$$
\begin{aligned}
& V_{i} K_{i}=\frac{\alpha_{2_{(i)}}^{2} y^{0, \ldots 0, \alpha_{1_{(i)}}, 0, \ldots 0}(t)-\alpha_{1_{(i)}}^{2} y^{0, \ldots 0, \alpha_{2_{(i)}}, 0, \ldots 0}(t)}{\alpha_{1_{(i)}}^{2}+\alpha_{2_{(i)}}^{2}}, \\
& V_{i i} K_{i i}=\frac{y^{0, \ldots 0, \alpha_{1_{(i)}}, 0, \ldots 0}(t)+y^{0, \ldots 0, \alpha_{2_{(i)}}, 0, \ldots 0}(t)}{\alpha_{1_{(i)}}^{2}+\alpha_{2_{(i)}}^{2}} .
\end{aligned}
$$

For terms containing non-symmetric kernels, we supply signals with amplitudes $\alpha_{1_{(i)}}$ and $\alpha_{1_{(j)}}(j \neq i)$. Signals whose numbers differ from $i$ and $j$ are set equal to zero. Thus, we have

$$
\begin{aligned}
& \alpha_{1_{(i)}} \cdot V_{i} K_{i}+\alpha_{1_{(i)}}^{2} V_{i i} K_{i i}+\alpha_{1_{(j)}} \cdot V_{j} K_{j}+\alpha_{1_{(j)}}^{2} V_{j j} K_{j j}+ \\
& +\alpha_{1_{(i)}} \cdot \alpha_{1_{(j)}} V_{j i} K_{j i}=y^{0, \ldots 0, \alpha_{1_{(j)}}, 0, \ldots 0, \alpha_{1_{(i)}}, 0, \ldots 0}(t),
\end{aligned}
$$

and, taking into account (8), we obtain

$$
\begin{aligned}
& \alpha_{1_{(i)}} \cdot V_{i} K_{i}+\alpha_{1_{(i)}}^{2} V_{i i} K_{i i}+\alpha_{1_{(j)}} \cdot V_{j} K_{j}+\alpha_{1_{(j)}}^{2} V_{j j} K_{j j}+ \\
& +\alpha_{1_{(i)}} \cdot \alpha_{1_{(j)}} V_{j i} K_{j i}=y^{0, \ldots 0, \alpha_{1_{(j)},}, 0, \ldots, \ldots 0, \alpha_{1_{(i)}}, 0, \ldots 0}(t), \\
& V_{j i} K_{j i}=\frac{1}{\alpha_{1_{(i)}} \cdot \alpha_{1_{(j)}}}\left(y^{0, \ldots 0, \alpha_{1_{(j)}}, 0, \ldots 0, \alpha_{1_{i j},}, 0, \ldots 0}(t)-\right. \\
& \left.-\alpha_{1_{(i)}} \cdot V_{i} K_{i}-\alpha_{1_{(i)}}^{2} V_{i i} K_{i i}-\alpha_{1_{(j)}} \cdot V_{j} K_{j}-\alpha_{1_{(j)}}^{2} V_{j j} K_{j j}\right) .
\end{aligned}
$$

The number of all series of signals for terms containing non-symmetric kernels is $C_{p}^{2}$.

For $N=3$ we do the same. Omitting cumbersome calculations, we find that the number of signal series for the terms containing symmetric kernels is $3 p$, the number of all series of signals for terms containing partially symmetric kernels is $3 C_{p}^{2}$ and the number of all series of signals for terms containing non-symmetric kernels is $C_{p}^{3}$.

Move on to the second stage. Suppose that the method of middle rectangles is used to construct a discrete model, where $n$ is the number of nodes of a uniform mesh.

Based on the assumptions made, in case $N=2$, we have $n$ unknowns for terms containing a onedimensional kernel, and $p$ terms themselves. We have $n(n+1) / 2$ unknowns for the terms containing a twodimensional symmetric kernel and $p$ terms themselves, as in the one-dimensional case. 
In the term containing the non-symmetric kernel, due to the lack of symmetry, the number of unknowns is $n^{2}$, and the number of signals supplied is $C_{p}^{2}$. Thus, the required number of initial data for $N=2$ is

$$
\mathrm{S}_{2}=2 p\left(p n+\frac{p n(n+1)}{2}\right)+C_{p}^{2} \cdot C_{p}^{2} n^{2} .
$$

For $N=3$, the first two terms are defined in a similar way as for $N=2$. Figure 5 will help to determine the number of unknowns for terms containing three-dimensional integrals.

In the case of symmetry, the domain of definition is divided into six parts and it is enough to determine the number of nodes in one of these parts. Fig. 5 show that the required number of unknowns, in this case, is defined as $\frac{n(n-1)(n-2)}{6}$. The number of terms with threedimensional integrals is $p$.
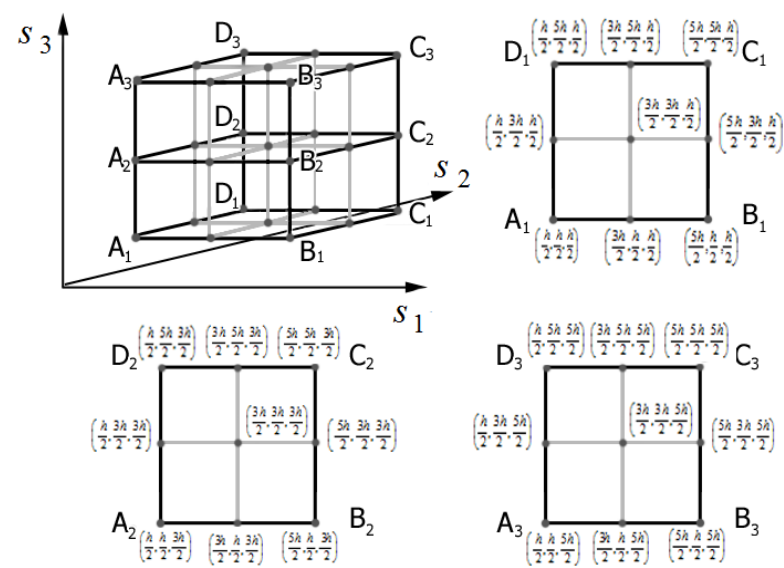

Fig. 5. Domain of definition of three-dimensional kernels at $n=3$.

In the case of partial symmetry, the domain of definition is divided into three subdomains of the same volume. The number of nodes of one such subdomain is $\frac{n^{2}(n+1)}{2}$. The number of terms containing partially symmetric kernels is $p(p-1)$.

Thus, in a term containing the non-symmetric kernel, due to the lack of symmetry, the number of unknowns is $n^{3}$, and the number of such terms is $C_{p}^{3}$. Therefore, the required number of initial data is found as

$$
\begin{aligned}
& \mathrm{S}_{3}=3 p\left(p n+\frac{p n(n+1)}{2}+p \frac{n(n-1)(n-2)}{6}\right)+ \\
& +3 C_{p}^{2} \cdot C_{p}^{2} n^{2}+3 C_{p}^{2} \cdot p(p-1) \frac{n^{2}(n+1)}{2}+C_{p}^{3} \cdot C_{p}^{3} n^{3} .
\end{aligned}
$$

\section{Conclusions}

The work discusses the applicability of the technique using the representation of a dynamic object in the form of a segment of the Volterra series for constructing a vector model. The segment of the steam-water path of the power unit of the Nazarovo power station is considered as an example. The time to reach the stationary mode was analyzed and recommendations for choosing the length of the time interval were obtained for input disturbances of a special type. The analysis of the complexity of applying the $\mathrm{m}$ technique presented in the work is carried out. The number of unknowns necessary for constructing a mathematical model is obtained in the case of a vector input disturbance with an arbitrary dimension for the quadratic and cubic segments of the Volterra series.

The research was carried out under State Assignment, Project 17.3.1 (Reg. No. AAAA-A17-117030310442-8) and Project 17.1.3 (Reg. No. AAAA-A17-117030310443-5) of the Fundamental Research of Siberian Branch of the Russian Academy of Sciences.

\section{References}

1. N.N. Bakhtadze, V.A. Lototsky, E.M. Maksimov, N.E. Maksimova, Information technology and computing systems, 3, 45 (2011)

2. A.Z. Gamm, I.N. Kolosok, R.A. Zaika, Elektrichestvo, 10, 2 (2005)

3. V.G. Kurbatskiy, N.V. Tomin, Systems. Methods. Technologies, 1(1), 113, (2009)

4. V.G. Khapusov, A.A. Ermakov, A.A. Podkorytov, Proceedings of ISTU, 22 (3), 112 (2018)

5. S. Solodusha, V. Spiryaev, E. Tairov, Proc. XXI International Conference "Complex Systems: Control and Modeling Problems" (CSCMP), 616 (2019)

6. E.D. Antipina, V.A. Spiryaev, E.A. Tairov, E3S WEB of Conferences, 114, 01007 (2019)

7. V. Volterra, A Theory of Functionals, Integral and Integro-Differential Equations (Nauka, Moscow, 1982)

8. A. Apartsyn, Nonclassical Linear Volterra Equations of the First Kind (VSP, Utrecht-Boston, 2003)

9. S. Solodusha, Methods for constructing integral models of dynamic systems: algorithms and applications in power engineering (Irkutsk, ESI SB RAS, 2019)

10. E.A. Tairov, A.A. Loginov, V.F. Chistyakov, Mathematical model, numerical methods and software of the simulator for the generating unit of the Irkutsk CHP-10 (Irkutsk, ESI SB RAS, Preprint № 11, 1999)

11. A.S. Apartsyn, Proc. XII Baikal International Conf. "Methods of Optimization and their applications", 1, 68 (2001) 